\title{
Study on Surface Electric Field Simulation of High Voltage Transmission Line Assembly
}

\author{
Liankai Chen1, Wenqing Lai', Jun Wang², Guoyi Jiang1, Yan Zhou1, Yong Chen², \\ Haibo Liu1, Zhaoyu Qin², Lei Ke ${ }^{2}$, Lei Wang2, Yang Shen² \\ ${ }^{1}$ East Inner Mongolia Electric Power Company Limited, Inner Mongolia, Hohhot, China \\ ${ }^{2}$ Wuhan NARI Limited Liability Company of State Grid Electric Power Research Institute, Wuhan, China \\ Email: chenliankai@md.sgcc.com.cn
}

Received September 2013

\begin{abstract}
For the studies in the field of high voltage power transmission, this paper has adopted the method of finite element node potential, and put forward two kinds of high pressure sensor-fixture modeling scheme for the sensor-fixture of the high voltage side, the simulation analysis shows that the sensor-fixture surface should be smooth, and should not appear the conclusion of edges and corners. While through establishing the four clamps assembly optimized model, and simulates the strain gages, fixtures and conductor surface field strength and electric field distribution in the model as a whole in turn, this paper Finally got the optimal size of fixture structure and assembly of each part reasonable location layout.
\end{abstract}

\section{Keywords}

High Voltage Assembly; Finite Element Method; Simulation Calculation; Electric Field Distribution

\section{Introduction}

Transmission line is the material basis for the development of electric power industry. The transmission grid facilities are burdened with the task of transmission and distribution of electrical energy, the reliability and operating conditions of which directly determine the stability and security of the entire system, but also determine the power quality and reliability of supply. Currently, with the difficult inspection and heavy maintenance workload, if the problems on the transmission line cannot be promptly found and solved, it will affect the country's production and residents' life, which has become an imperative social problem that ensures the safety and smooth flow of the power "lifeline" [1].

With the increasing of the grid capacity and transmission voltage rating, it's becoming increasingly important that the on-line monitoring of the performance of the high voltage power transmission equipment in operating [2].

\section{Calculation Principle}

\subsection{Finite Element Analysis of Electrostatic Field}

At present, the electromagnetic field numerical calculation method commonly used at home and abroad is the

How to cite this paper: Chen, L.K., Lai, W.Q., Wang, J., Jiang, G.Y., Zhou, Y., Chen, Y., Liu, H.B., Qin, Z.Y., Ke, L., Wang, L. and Shen, Y. (2014) Study on Surface Electric Field Simulation of High Voltage Transmission Line Assembly. Journal of Power and Energy Engineering, 2, 554-563. http://dx.doi.org/10.4236/jpee.2014.24075 
charge simulation method of finite element method, boundary element method, finite difference method, and a variety of methods of coupling or mixed application method etc. [3].

Calculation of the model in this study is composed of a variety of media, and the model is more complex, which can be calculated by finite element method Finite element method based on variation principles, combined with the Difference method and developed a numerical method.

Electrostatic field potential energy can be expressed as a function and its derivative pending integral of the integration region (or solving the field), in accordance with the differential method of discrete method, it is divided into finite sub-regions (called cells). And then use these discrete units, the electrostatic field energy be approximated by a finite number of nodes in the potential of the function [4]. Thus, the electrostatic field energy demand is reduced to the problem of extreme multi-function extremum problem, which usually boils down to a set of multivariate linear algebraic equations finite element equation. Finally, the specific characteristics of equations, using appropriate algebraic methods, and seek each node potential, to achieve a discrete solution of variation problems.

In the electrostatic field, generally solving potential of $\varphi$ is a direct object. In isotropic, linear, homogeneous medium, the potential to meet the Poisson equation or the Laplace equation:

$$
\nabla^{2} \varphi=\frac{\rho}{\varepsilon}
$$

Or

$$
\nabla^{2} \varphi=0
$$

where, $\rho$ is free charge density, $\varepsilon$ is the dielectric constant.

Based on the principle of least action electrostatic field, in the medium a fixed charge system, the surface charge distribution of the electric field tends to always make synthesis with minimal electrostatic energy [5].

The entire field for a given potential values on the boundary of the first class of boundary value problems, energy integral expression is:

$$
W_{e}=\iint_{D}\left\{\frac{\varepsilon}{2}\left[\left(\frac{\partial \varphi}{\partial x}\right)^{2}+\left(\frac{\partial \varphi}{\partial y}\right)^{2}\right]-\rho \varphi\right\} d x d y d z=\min
$$

Finite element method is a continuous field via split into a finite number of discrete units, in two-dimensional field problems commonly used to split triangular and quadrilateral elements, within each cell of the potential function approximated by polynomial interpolation.

After the split, the secondary functional can be expressed as the sum of all the cells energy function:

$$
\mathrm{F}(\varphi)=\sum_{e=1}^{q} \mathrm{~F}_{e}(\varphi)
$$

In the formula, $\mathrm{F}(\varphi)$ represents the element $\varepsilon$ of the corresponding triangular energy integral, which is a unit of energy integration [6]:

$$
\mathrm{F}_{e}(\varphi) \approx \mathrm{F}_{e}\left(\varphi^{e}\right)=\iint_{D} \frac{\varepsilon}{2}\left[\left(\frac{\partial \varphi^{e}}{\partial x}\right)^{2}+\left(\frac{\partial \varphi^{e}}{\partial y}\right)^{2}\right] d x d y=\min
$$

While:

$$
\varphi^{e}=\sum_{i=1}^{q} N_{i}^{e} \varphi_{i}^{e}
$$

Then:

If $\frac{\partial N_{i}^{\varepsilon}}{\partial x}$ is independent of $x$, then:

$$
\iint_{D} \frac{\varepsilon}{2}\left(\frac{\partial \varphi^{e}}{\partial x}\right)^{2} \mathrm{~d} x \mathrm{~d} y=\iint_{D} \frac{\varepsilon}{2}\left(\sum_{i=1}^{q} \frac{\partial N_{i}^{e}}{\partial x} \varphi_{i}^{e}\right)^{2} \mathrm{~d} x \mathrm{~d} y
$$




$$
\iint_{D} \frac{\varepsilon}{2}\left(\frac{\partial \varphi^{e}}{\partial x}\right)^{2} \mathrm{~d} x \mathrm{~d} y=\varphi^{e \mathrm{~T}} \boldsymbol{K}_{x}^{e} \varphi^{e}
$$

Of which:

$$
\begin{aligned}
& \varphi^{e}=\left[\begin{array}{c}
\varphi_{1}^{e} \\
\vdots \\
\varphi_{q}^{e}
\end{array}\right] \\
& \boldsymbol{K}_{x}=\frac{\varepsilon S^{e}}{2}\left[\begin{array}{cccc}
\frac{\partial N_{1}}{\partial x} \frac{\partial N_{1}}{\partial x} & \frac{\partial N_{1}}{\partial x} \frac{\partial N_{2}}{\partial x} & \cdots & \frac{\partial N_{1}}{\partial x} \frac{\partial N_{q}}{\partial x} \\
\frac{\partial N_{2}}{\partial x} \frac{\partial N_{1}}{\partial x} & \frac{\partial N_{2}}{\partial x} \frac{\partial N_{2}}{\partial x} & \cdots & \frac{\partial N_{2}}{\partial x} \frac{\partial N_{q}}{\partial x} \\
\vdots & \vdots & \ddots & \vdots \\
\frac{\partial N_{q}}{\partial x} \frac{\partial N_{1}}{\partial x} & \frac{\partial N_{q}}{\partial x} \frac{\partial N_{2}}{\partial x} & \cdots & \frac{\partial N_{q}}{\partial x} \frac{\partial N_{q}}{\partial x}
\end{array}\right]
\end{aligned}
$$

$S^{e}$ is the split region enclosed area. Similarly, if $\frac{\partial N_{i}^{\varepsilon}}{\partial y}$ has nothing to do with y, then:

$$
\begin{gathered}
\left(\frac{\partial \varphi^{e}}{\partial y}\right)^{2}=\left(\sum_{i=1}^{q} \frac{\partial N_{i}^{e}}{\partial y} \varphi_{i}^{e}\right)^{2}=\varphi^{e T} \boldsymbol{K}_{y}^{e} \varphi^{e} \\
\boldsymbol{K}_{y}=\frac{\varepsilon S^{e}}{2}\left[\begin{array}{cccc}
\frac{\partial N_{1}}{\partial y} \frac{\partial N_{1}}{\partial y} & \frac{\partial N_{1}}{\partial y} \frac{\partial N_{2}}{\partial y} & \cdots & \frac{\partial N_{1}}{\partial y} \frac{\partial N_{q}}{\partial y} \\
\frac{\partial N_{2}}{\partial y} \frac{\partial N_{1}}{\partial y} & \frac{\partial N_{2}}{\partial y} \frac{\partial N_{2}}{\partial y} & \cdots & \frac{\partial N_{2}}{\partial y} \frac{\partial N_{q}}{\partial y} \\
\vdots & \ddots & \vdots \\
\frac{\partial N_{q}}{\partial y} \frac{\partial N_{1}}{\partial y} & \frac{\partial N_{q}}{\partial y} \frac{\partial N_{2}}{\partial y} & \cdots & \frac{\partial N_{q}}{\partial y} \frac{\partial N_{q}}{\partial y}
\end{array}\right]
\end{gathered}
$$

Therefore:

$$
\mathrm{F}_{e}\left(\varphi^{e}\right)=\varphi^{e \mathrm{~T}}\left(\boldsymbol{K}_{x}^{e}+\boldsymbol{K}_{y}^{e}\right) \varphi^{e}
$$

All sub-regions combined simultaneous equations, we obtain:

$$
\mathrm{F}(\varphi) \approx \varphi^{\mathrm{T}} \boldsymbol{K} \varphi
$$

where $\varphi$ and $\mathrm{K}$ is the set of simultaneous equations after all the variables and coefficient matrix. So the problem is discretized into functional multivariate quadratic function extremum problem:

$$
\varphi^{\mathrm{T}} \boldsymbol{K} \varphi=\min
$$

By the function extreme value theory, we have:

$$
\frac{\partial \mathrm{F}}{\partial \varphi_{i}}=0
$$

The formula is:

$$
K \varphi=0
$$

The above equation is the finite element equations, which can solve the equation by using the boundary conditions; and you can get the desired approximate solution.

In summary, the finite element method calculation steps are as follows:

1) Clear field range, the field split into a finite element (two-dimensional field can be trilateral or quadrilateral 
base unit, three-dimensional field available tetrahedral or hexahedral basic unit);

2) Electric energy calculation unit elements of the coefficient matrix;

3) Calculate the total electric energy coefficient matrix elements;

4) Finite element equations are listed;

5) Solving the finite element equations, find the node potential;

6) Potential can be calculated based on each node's remaining amount of the electric field.

\subsection{ANSYS Software in the Application of the Electrostatic Field Calculation}

ANSYS is the financial structure, fluid, electric, magnetic, acoustic analysis in one large general-purpose finite element analysis software. Its electromagnetic field analysis includes several modules: low frequency, high frequency, high frequency TV size (MOM), cable bundle electromagnetic compatibility (EMC) and signal integrity (SI), PCB's and other EMC and SI [7]. It works with most CAD software interface, data sharing and exchange, such as Pro/Engineer, AutoCAD, Solid works, etc.

ANSYS analysis process consists of three main steps: pre-treatment (create or read into the finite element model, defining material properties, mesh); loading and solving (applied load and set constraints solving); post-treatment (see Analysis a result, the check result is correct) [8].

Among them, the pre-treatment of the mechanical structure of the system is mainly made-up of nodes and elements into the mix into a finite element model of the various parts of its defined element type, material properties and real constants, then meshing, finite element model to obtain the unknown nodes and elements. Loading and Solution mainly defined properly, the correct load system components in order to understand the structure after the reaction by the external load, load on the finite element model, then select the appropriate direct solver and iterative solver for solving computing nodes [9]. The subsequent processing is mainly analysis node inspection and unit results.

\section{Calculation Models and Parameters Established}

\subsection{Research Contents}

In order to effectively avoid the transmission line conductor aeolian vibration caused by the wire off shares accidents, it requires real-time monitoring of wire in the breeze under the vibration information, so need to install the wire strain measuring device measuring wire strain. Strain gauge the middle part of the metal, composite material with metal wire ends, fixture and fixed with strain gage. Strain gauge on the wire installation diagram as shown in Figure 1. Different structure of the metal fixture assembly surface field strength will produce different effects, so the need for fixture structure modeling of different options to select the optimal metal clamp structure to reduce vibration and point discharge breeze on high-voltage transmission wires resulting damage.

$\mathrm{n}$ this study, a high-pressure side sensor fixture design two programs, In order to verify the merits of two kind of fixture, respectively, finite element analysis of the phenomenon of charge concentration of two kinds of fixture scheme. The following is the detailed modeling program.

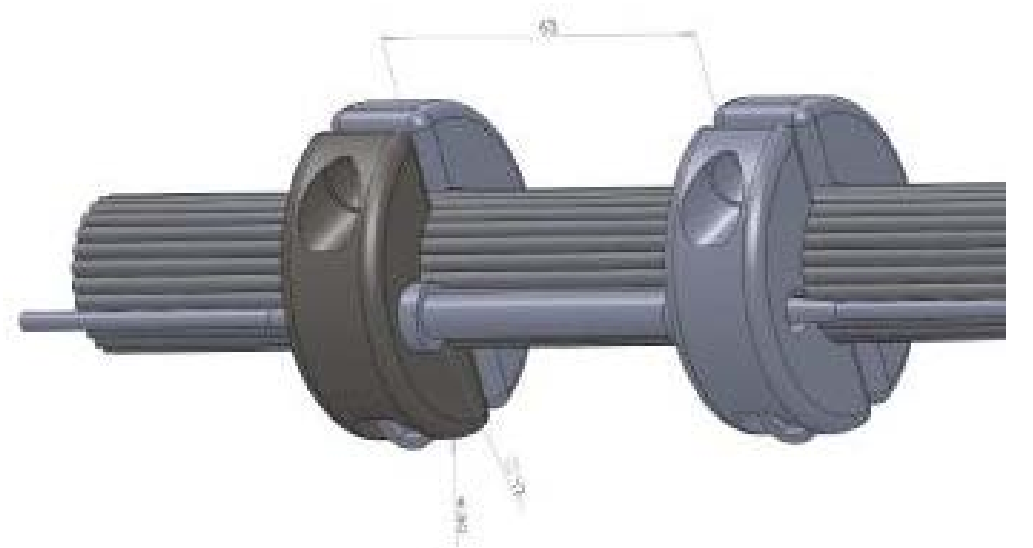

Figure 1. Strain gauge assembly simulation diagram. 


\subsection{Sensor Fixture Modeling}

The surface of the clamp tip scheme I will have the electric field concentration, and easily lead to burn out the wire tip discharge, while the scheme II uses the methods to avoid the tip, the fixture body with circular arc design, the design of bolt connection parts of sinking, completely hidden in the fixture screw bolt, Compact structure design of high voltage side as shown in Figure 2.

Since two briquetting symmetrical structure and coupling docking, we are concerned about the charge distribution is mainly concentrated in the outer surface of the arc. To save modeling time is taken in the right briquetting COMSOL modeling and analysis, shown in Figure 3. Similarly, removal of the air domain compact structure only concerned with compact contour of the air within the inner boundary of the electric field distribution. The simulation parameters are shown in Table 1.

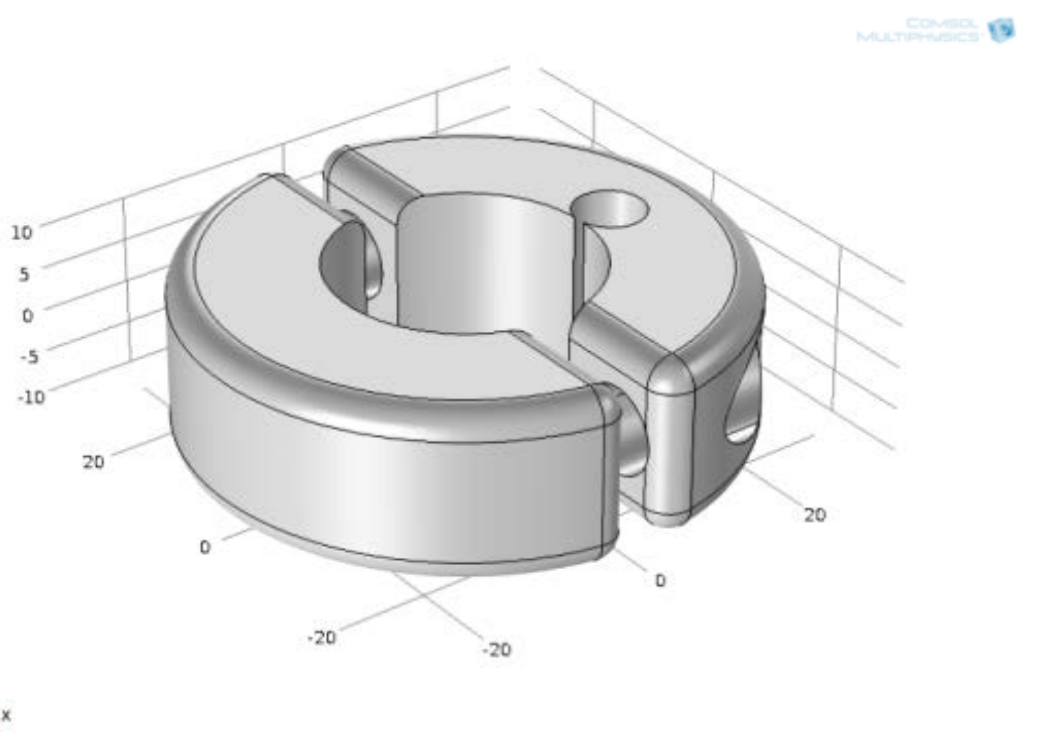

Figure 2. Compact structure design of high voltage.

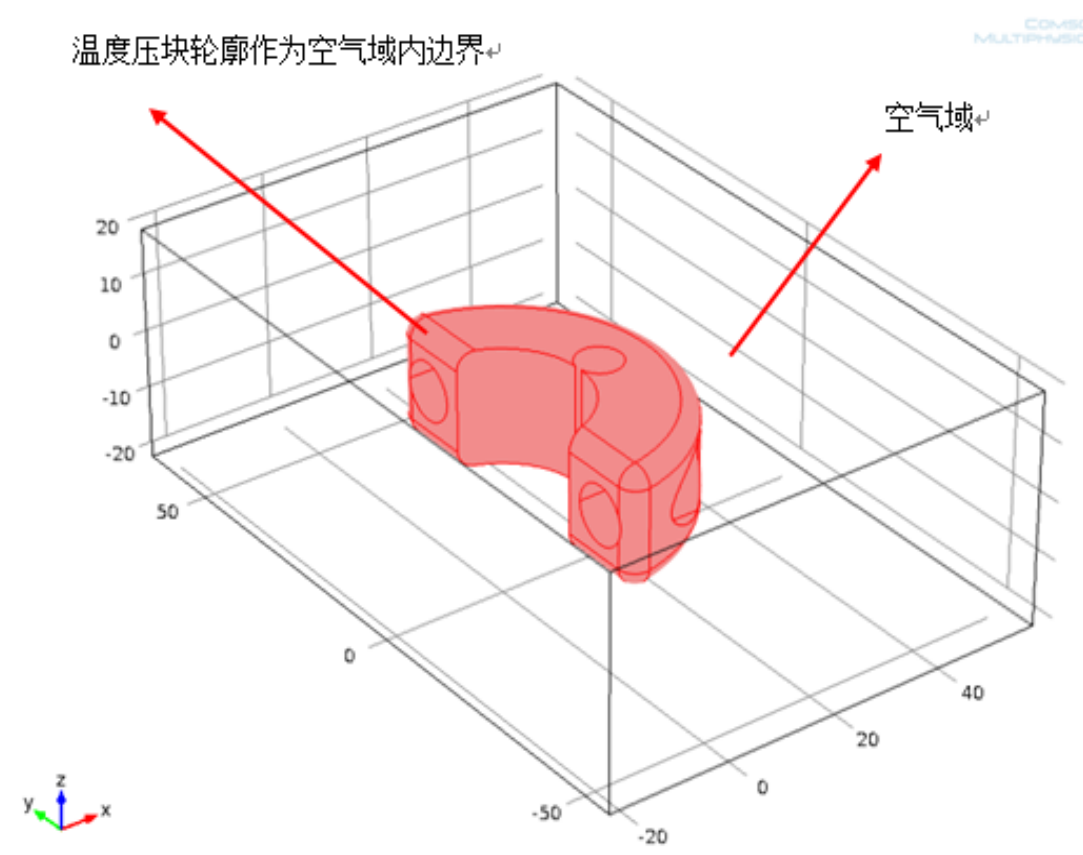

Figure 3. The compacts parcels by air domain. 
Table 1. Scheme of B simulation parameter list.

\begin{tabular}{cc}
\hline simulation parameter & Parameter values \\
\hline $\begin{array}{c}\text { Geometric parameters } \\
\text { Material parameters }\end{array}$ & $\begin{array}{r}\text { With length } 80 \mathrm{~mm} \text {, width } 120 \mathrm{~mm}, 60 \mathrm{~mm} \text { high air field surrounded fixture. } \\
\text { Air domain and the pressing block center symmetry. } \\
\text { air }\end{array}$ \\
Beshing & $\begin{array}{r}\text { Using user-controlled mesh, the largest unit length } 2 \mathrm{~mm} \text {, the smallest unit of length } 1 \mathrm{~mm}, \\
\text { cell growth rate of } 1.3 . \text { Finally mesh degrees of freedom } 1,854,100 .\end{array}$ \\
Fixture inner contour of the air domain boundary, surface potential $220 \mathrm{kV}$, \\
air domain boundaries are deemed to be grounded.
\end{tabular}

\section{Results and Analysis}

\subsection{Analysis of Sensor Modeling Scheme I of Fixture Results}

A sensor jig modeling scheme potential distribution of the simulation results shown in Figure 4, we can clearly see the outline edge clamps outwardly from the $220 \mathrm{kV}$ to zero potential (ground) of the uniform field in the ambient air.

The electric field distribution simulation results shows that the electric field concentration at the tip of the metal clamp surface corner. The surface potential of $220 \mathrm{kV}$ case, the tip surface of the field about $5.32 \times 10^{7}$ $\mathrm{v} / \mathrm{m}$, and in the circular surface is uniform electric field distribution. So, in order to avoid the discharge temperature and humidity in the extreme case, fixture surface should be smooth.

\subsection{Analysis of Sensor Modeling Scheme II of Fixture Results}

The results of simulation of air field potential distribution scheme of II as shown in Figure 5, potential along the air domain boundary from $220 \mathrm{kV}$ to 0 steady decline, and the scheme of simulation results similar to I.

The electric field distribution simulation results as shown in Figure 8, the electric field intensity around the right arc is about $1.81 \mathrm{e}^{7}-1.87 \mathrm{e}^{7} \mathrm{~V} / \mathrm{m}$. It is much lower than the electric field of a rectangular boundary scheme of fixture design focus on the value, and the uniform electric field distribution, no surge spikes.

Comparison of the simulation results of plan I and II, the electric field concentration at the tip of the metal clamp surface corner. The surface potential of $220 \mathrm{kV}$ case, the tip surface of the electric field is about $5.32 \times$ $10^{7} \mathrm{v} / \mathrm{m}$, and in the circular surface is uniform electric field distribution. Peak electric arc chamfer is about $2.18 \mathrm{e}^{7} \mathrm{~V} / \mathrm{m}-2.36 \mathrm{e}^{7} \mathrm{~V} / \mathrm{m}$, and the rectangular clamp tip electric field peak compared to $5.32 \mathrm{e}^{7} \mathrm{~V} / \mathrm{m}$ is reduced by about $50 \%$, and the boundary of uniform electric field distribution. Arc pressing block is not easy to generate point discharge, optimization design.

In order to avoid the discharge temperature and humidity in the extreme case, fixture surface should be smooth [10]. The optimization scheme is to use arc chamfering, and as far as possible to ensure that the surface finish. Give extended application, low end fixture, especially from the wire clamp close, in order to avoid the tip electric caused by induction, the edges and corners also need passivation [11].

\subsection{Results and Analysis of the Electric Field Distribution Simulation Model Assembly}

This study is based $220 \mathrm{kV}$ transmission line of the finite element model was established by the four kinds of mode, Model A is the original model, model B is the only changes bolt model, model C in the second model, based on the composite metal and the sensor connection portion is placed jig hole, model $\mathrm{D}$ is changed rounded $0.8 \mathrm{~mm}$ bolt hole wall model, outsourcing air cylinder modeled by a radius of $100 \mathrm{~m}$.

Using ANSYS software based on a section of the model diagram of four models for the combination of data modeling. Model A is the original model; model B is changed bolt model; C model based on B model, the sensor metal and composite joints on the fixture hole; model of $\mathrm{D}$ is modified bolt hole wall fillet $0.8 \mathrm{~mm}$ model; the overall distribution of the electric field then the simulation of the four types of models, the whole field distribution results are shown in Figures 6-9.

From this point of view of the whole electric field distribution, models A and the electric field under the other three models are quite different, model B, model C and model D is close to. The maximum field intensity model A appeared on the ends of the bolts is $64.19 \mathrm{kV} / \mathrm{cm}$, maximum field strength, model B, model C and model D is 


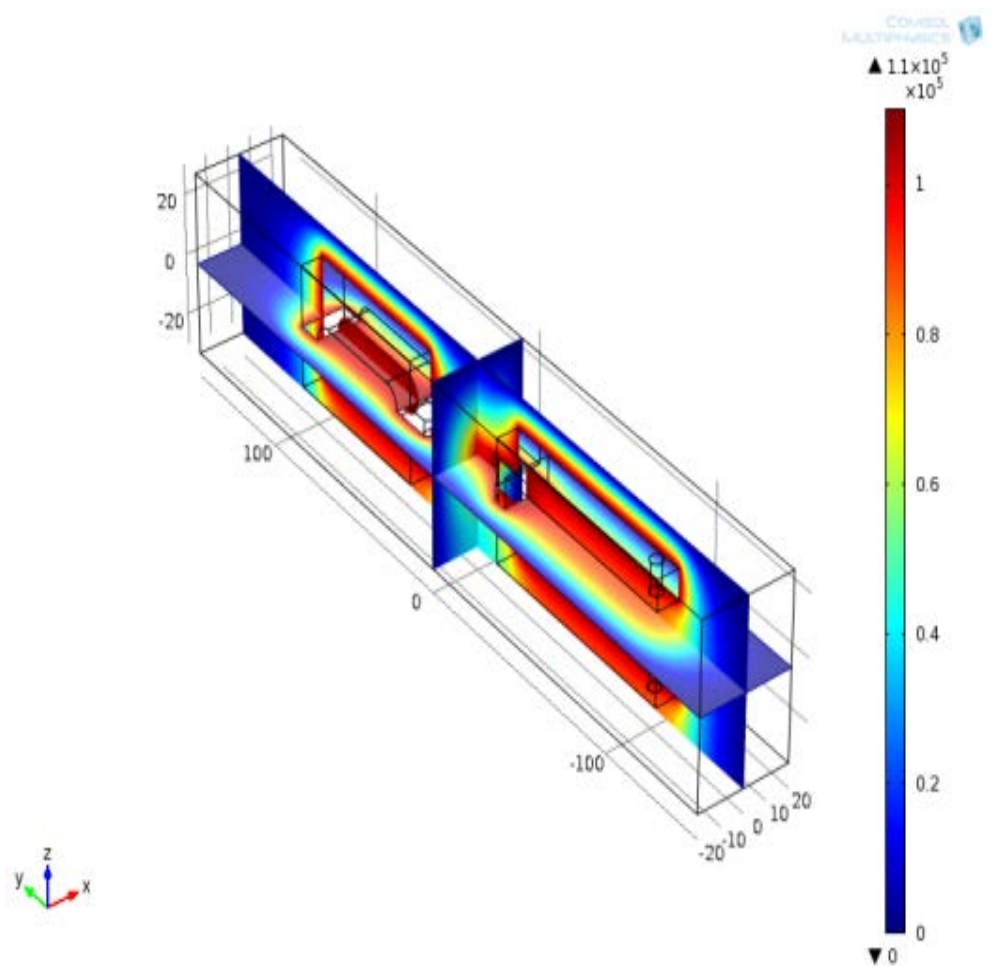

Figure 4. Distribution of the air domain potential surrounding the fixture in scheme I.

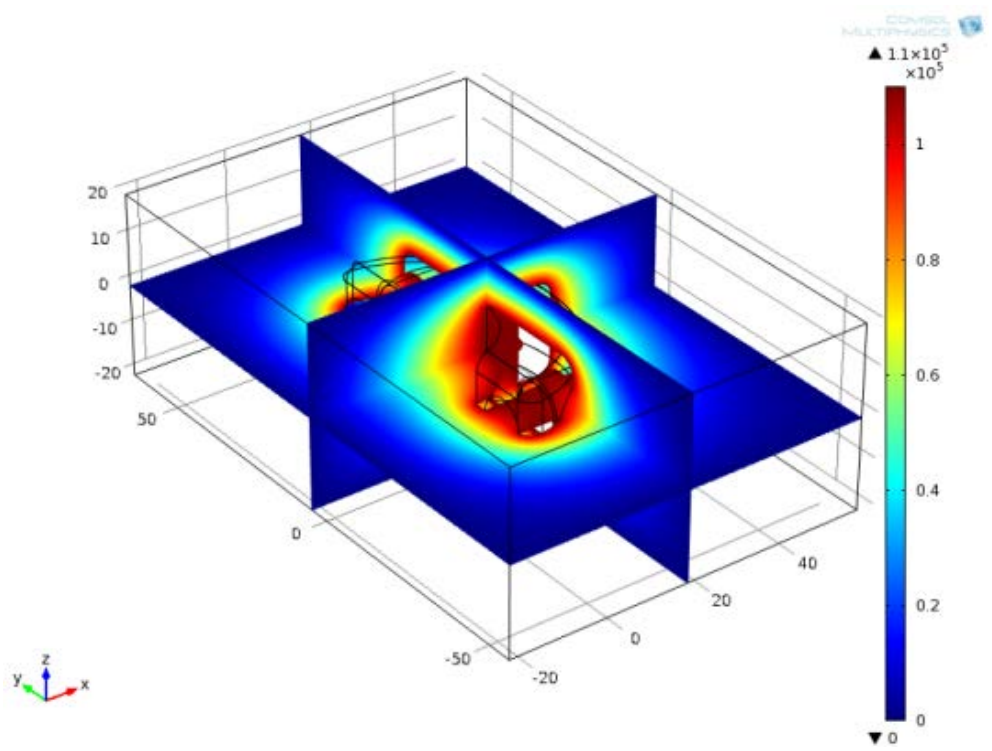

Figure 5. Distribution of the air domain potential surrounding the fixture scheme in B.

appeared in the top corner of fixture, the maximum electric field strength model for B $28.14 \mathrm{kV} / \mathrm{cm}$, the maximum field strength model for C $28.16 \mathrm{kV} / \mathrm{cm}$, the maximum field strength model of $\mathrm{D}$ is $28.26 \mathrm{kV} / \mathrm{cm}$, the maximum electric field strength of three kinds of model are very small.

In order to compare the difference between different models of the maximum field intensity, listed in Table 2 field in different parts of the different model maximum.

As can be seen from the Table 2, the potential of the composite section in both ends of strain gauge uneven 

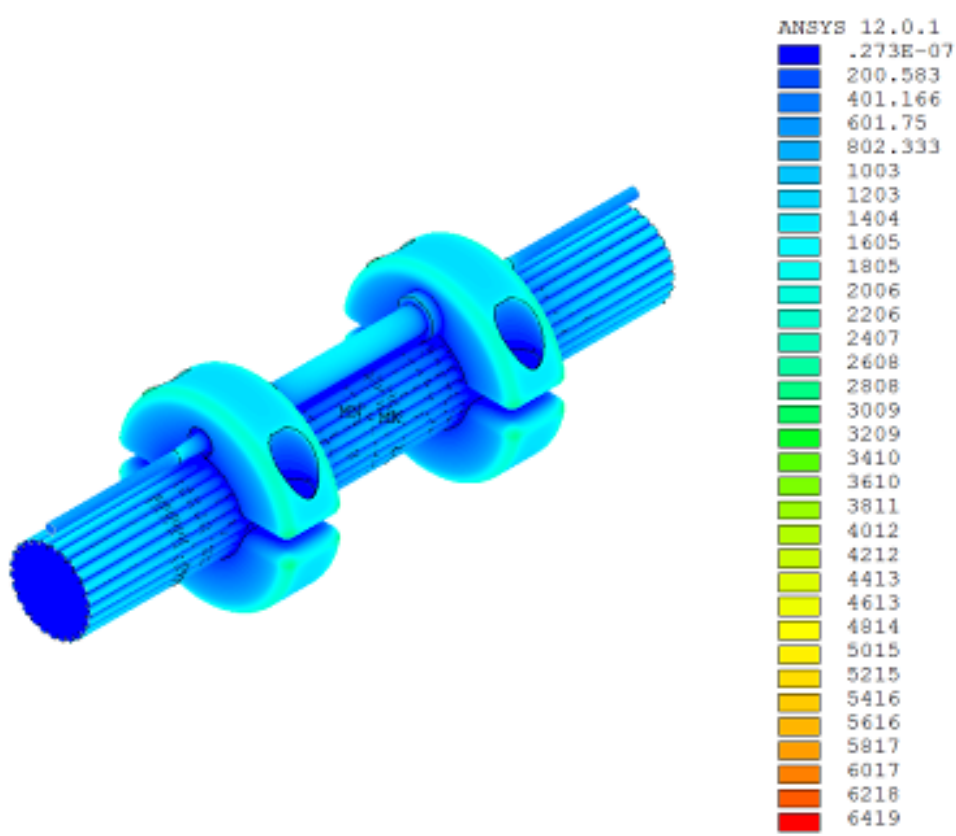

Figure 6. Model A whole field distribution.

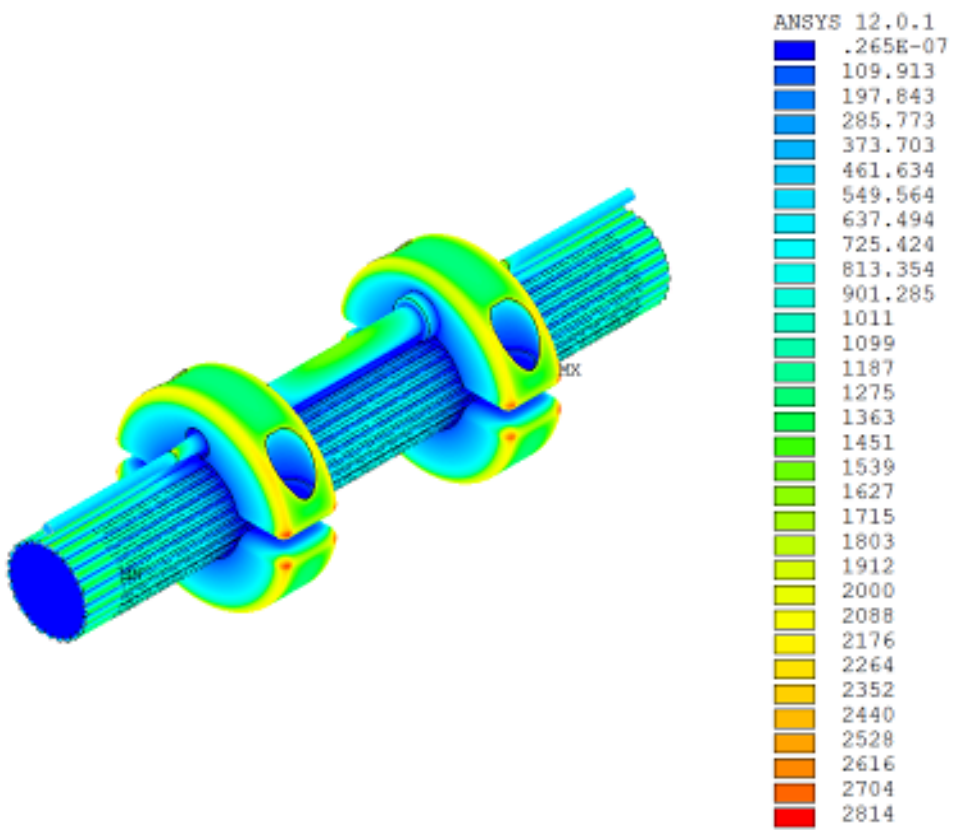

Figure 7. Model B whole field distribution.

Table 2. Different models of different parts of the maximum value.

\begin{tabular}{ccccc}
\hline & Model A & Model B & Model C & Model D \\
\hline The maximum electric field value $(\mathrm{kV} / \mathrm{cm})$ & 64.19 & 28.14 & 28.16 & 28.26 \\
Conductor surface field maximum $(\mathrm{kV} / \mathrm{cm})$ & 15.77 & 15.74 & 15.77 & 15.82 \\
Clamp the maximum surface electric field $(\mathrm{kV} / \mathrm{cm})$ & 27.64 & 28.14 & 28.06 & 28.26 \\
The maximum strain gage surface electric field $(\mathrm{kV} / \mathrm{cm})$ & 21.13 & 21.75 & 16.65 & 15.94 \\
\hline
\end{tabular}



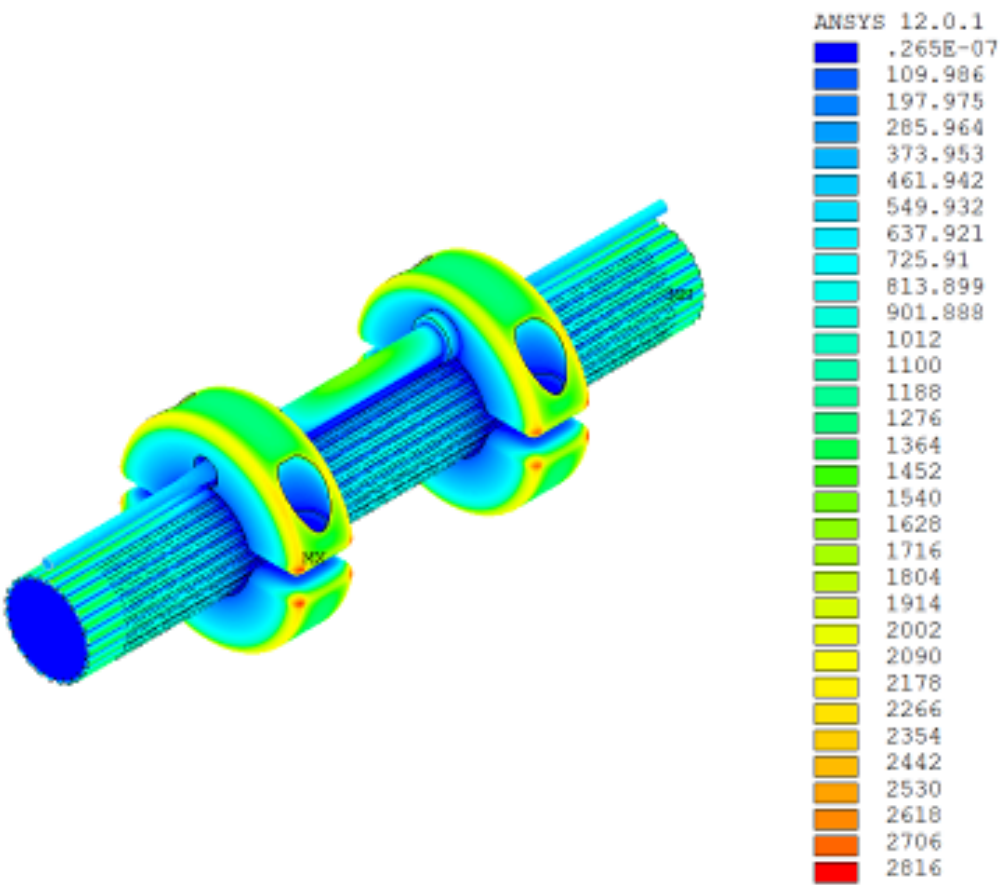

Figure 8. Model C whole field distribution.

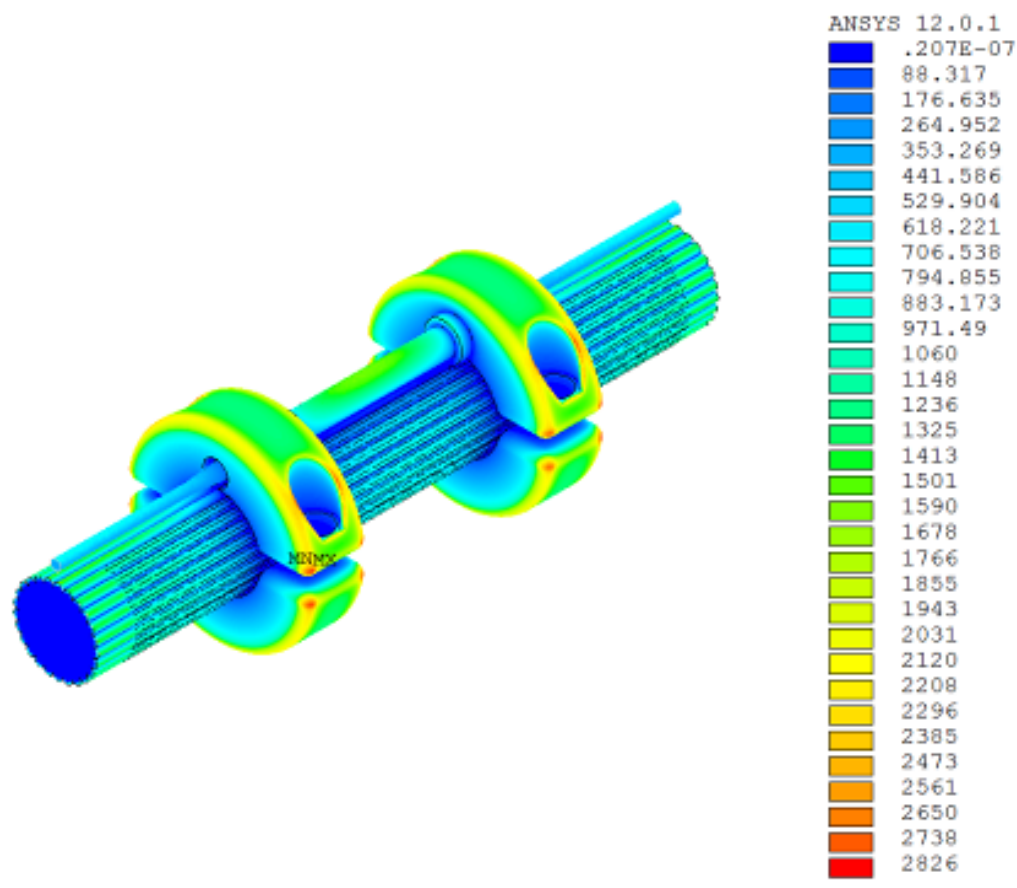

Figure 9. Model D whole field distribution.

distributed in the four models. The potential is highest at the part connect with the metal, and lowest at both ends. The overall integral maximum field value in model one was significantly higher than the other three models. At the same time, the maximum field strength value of strain gauges in model one and model two was greater than the other two models. Comprehensive comparison of the four models, proposed to shorten the clamp bolt to place the end portion inside the clamp in order to avoid excessive field strength appears. The fillet radius of clamp portion can be appropriately enlarged to reduce the surface field strength [12]. 


\section{Summary}

Through the simulation and analysis and comparison of the two modeling programs of sensor fixture, that the electric field distributed more concentrated at the corner of the tip of the metal clamp's surface, while it's more even at the arc surface. The surfaces of metal clamps should be as smooth as possible, do not appear at the angular [13], the optimal approach is to use curved chamfer, which can effectively avoid the discharge at the tips under the extreme temperature conditions. In the design of the model assembled, place the joints which connected with metal and composite material inside the clamps, to prevent the field strength is too large on the surface of the strain gauge. And the metal fixture can reduce its surface field strength by properly increasing the fillet radius, making the design of model assembly optimized.

\section{References}

[1] Wang, J.-Y., Fang, C.-E., Dai, Y.-S., Li, W. and Hou, X.-Y. (2007) Electric Field Calculation and Optimal Design of 35 kV Contactor Box. High Voltage Engineering, 33, 63-65.

[2] Zhang, Y.-J., Li, X., Liu, H.-B. and Sun, H.-H. (2012) Calculation and Optimization of Electric Field for $220 \mathrm{kV}$ Composite Hollow Insulators. North China Electric Power, 6, 16-19.

[3] Yang, Y. (2013) Calculation and Analysis of Total Electric Field at Ground Level beneath 800 kV DC Transmission Lines With Out-of-Operational Negative Pole Line. Power System Technology, 37, 1531-1535.

[4] Huang, D.C., Ruan, J.J., Chen, Y., Huo, F., Yu, S.F. and Liu, S.B. (2008) Study on the Voltage and Electric Field Distribution along Composite Insulator of $1000 \mathrm{kV}$ AC Transmission Line. Proceedings of the CSEE, 52-57.

[5] Li, Y. and Zhu, L.-X. (2013) Main Insulation Electric Field Calculation and Analysis Software for Power Transformer. Transformer, 50, 52-56.

[6] Jiang, X. and Wang, Z.Y. (2004) Calculation and Analysis for the Electric Field of Composite High Voltage Bushing. High Voltage Engineering, 30, 17-21.

[7] Lin, X., Cai, Q., Xu, J.-Y. and Li, S. (2011) Large Scale of Electric Field Calculation of 1100 kV Disconnector Based on Domain Decomposition Method. High Voltage Apparatus, 47, 1-6.

[8] Zhou, X.X., Lu, T.B., Cui, X., Zhen, Y.Z and Luo, Z.N. (2011) A Hybrid Method for the Simulation of Ion Flow Field of HVDC Transmission Lines Based on Finite Element Method and Finite Volume Method. Proceedings of the CSEE, 31, 127-133.

[9] Huang, D.-C., Ruan, J.-J. and Liu, S.-B. (2010) Potential Distribution along UHV AC Transmission Line Composite Insulator and Electric Field Distribution on the Surface of Grading Ring. High Voltage Engineering, 36, 1442-1447.

[10] Huang, D.-C., Wei, Y.-H., Zhong, L.-H., Ruan, J.-J. and Huang, F.-C. (2007) Discussion on Several Problems of Developing UHVDC Transmission in China. Power System Technology, 31, 6-12.

[11] Sun, C.-H., Zong, W., Li, S.-Q., Peng, Y.-H. and Ren, W.-W. (2006) A More Accurate Calculation Method of Surface Electric Field Intensity of Bundled Conductors. Power System Technology, 30, 92-96.

[12] Liu, G., Li, L., Zhao, X.J., Li, W.P., Li, B., Sun, Y.L., Ji, F. and Li, J.Z. (2012) Analysis of Nonlinear Electric Field of Oil-Paper Insulation under AC-DC Hybrid Voltage by Fixed Point Method Combined with FEM in Frequency Domain. Proceedings of the CSEE, 32, 154-161.

[13] Zhen, Y.Z., Cui, X., Luo, Z.N., Lu, T.B., Lu, J.Y., Yang, Y., Liu, Y.Q., Han, H. and Ju, Y. (2011) FEM for 3D Total Electric Field Calculation near HVDC Lines. Transactions of China Electrotechnical Society, 26, 153-160. 